\section{Sensibilisierung gegenüber Chinchilla bei Exposition im Haushalt}

Zusammenfassung: Der Kontakt mit Chinchillas im Haushalt kann zur Sensibilisierung und allergischen Rhinitis bei Kindern und Erwachsenen führen. Von sechs sensibilisierten Patienten mit ganzjähriger Rhinitis und/oder Asthma reagierten ein Kind und zwei Erwachsene positiv im nasalen Provokationstest mit Chinchillahaarextrakt. Allergenkarenz ist notwendig.

Sensibilization with Chinchilla on Exposition in Households: The contact to chinchilla in households may lead to sensibilization and allergic rhinitis in children and adults. In nasal provocation tests on 6 sensibilized patients suffering on perennial rhinitis and/or asthma one child and three adults showed positive reactions eith chinchilla hair extract. Allergen avoidance is recommended.

\section{Einleitung}

Tierhaare sind eine wichtige Quelle für Inhalationsallergene. In 37\% aller deutschen Haushalte werden Tiere gehalten [6]. Die Angaben über die Häufigkeit von Haustier-Allergien variieren aber stark je nach untersuchter Bevölkerungsgruppe und Diagnosekriterien. Fast 30\% aller Atopiker reagieren mit allergischen Sofortreaktionen auf Katzen- und Hundekontakt [5]. Eine schwedische Umfrage bei 40000 Schulkindern zeigte eine Häufigkeit von 5,3\% Haustier-Allergien [1]. In einer anderen schwedischen Studie an 110 asthmatischen Schulkindern betrug die Häufigkeit der Sensibilisierungen gegenüber Katzenepithelien 30\%, die gegenüber Hundehaaren 15\% [4].

Während die Häufigkeit von positiven Hauttesten gegenüber häufig gehaltenen Haustieren wie Hunden und Katzen gut untersucht ist, gibt es in der internationalen Literatur keine Angaben zur Sensibilisierung gegenüber Chinchillas. Überraschenderweise wird in Ratgebern in Zoohandlungen Allergikern von der Anschaffung der Tiere abgeraten [2].

Chinchillas erfreuen sich in den letzten Jahren als Heimtiere zunehmender Beliebtheit. Wegen ihres dichten und feinen Fells werden die aus Südamerika stammenden Tiere in großen Pelztierfarmen gezüchtet. Sie sind nachtaktiv und werden deshalb bevorzugt von Berufstätigen zu Hause gehalten. Die

Pneumologie 2000; 54: 373-374

(C) Georg Thieme Verlag Stuttgart · New York ISSN 0934-8387

\author{
G. Wesarg, K.-Ch. Bergmann \\ Allergie- und Asthma-Klinik, Bad Lippspringe
}

niedlichen, großäugigen Tiere eignen sich nicht als Kuscheltiere, auch wenn die Kinder sie attraktiv finden.

Die im Zoofachhandel erhältlichen Tiere sind Mischtypen aus Kurzschwanz- und Langschwanzchinchilla. Sie gehören zu den Nagetieren und sind mit dem Meerschweinchen, Nutria oder Eichhörnchen verwandt (Tab.1).

Tab. 1 Zoologische Einordnung des Chinchilla (Hasenmaus)

\begin{tabular}{ll}
\hline Ordnung & Nagetiere (Rodentia) \\
Unterordnung & Stachelschweinähnliche (Hystricomorpha) \\
Familie & Hasenmausartige (Chinchillidae) \\
Gattung & Chinchilla (Chinchilla) \\
Art & $\begin{array}{l}\text { Kurzschwanz-Chinchilla (Chinchilla chinchilla boli- } \\
\text { viana) Langschwanz-Chinchilla (Chinchilla laniera) }\end{array}$ \\
\hline
\end{tabular}

Wir prüften, ob bei Personen mit Chinchilla-Exposition im eigenen Haushalt Sensibilisierungen und allergische Rhinokonjunktivitiden auftreten.

\section{Patienten und Methode}

Untersucht wurden 6 Patienten, die sich wegen ganzjähriger Rhinokonjunktivitis oder Asthma bronchiale in der Ambulanz vorstellten und anamnestisch zu Hause mit Chinchillahaaren exponiert waren. Es wurden Zeitpunkt und Umstände der Anschaffung und Anzahl der Tiere, Ort der Tierhaltung, Freilauf im Zimmer und die Nikotinexposition in der Wohnung erfragt. Der Atopiestatus der Patienten wurde mit Hilfe der Familienanamnese, Pricktestung und Gesamt-IgE-Bestimmung (PRIST) erhoben. Die Pricktestung führten wir mit Chinchillaepithelienextrakt der Firma MASER durch; als positiv galt ein Quaddeldurchmesser über $5 \mathrm{~mm}$. Im Nasentest mit Rhinomanometrie wurde die klinische Aktualität der Sensibilisierungen gegenüber Chinchilla eruiert.

Allen Patienten gaben wir die Empfehlung, die Haustiere abzuschaffen. Nach einem Jahr wurden die Patienten gefragt, ob sie noch Chinchillas in der Wohnung halten.

\section{Ergebnisse}

In der Pricktestung wurde bei 2 Kindern (5 und 10 J.) und 4 Erwachsenen (20 bis $30 \mathrm{~J}$.) eine Sensibilisierung gegenüber Chinchillaepithelien nachgewiesen. Die Familienanamnese 


\begin{tabular}{|c|c|c|c|c|c|}
\hline $\begin{array}{l}\text { Alter, } \\
\text { Geschlecht }\end{array}$ & $\begin{array}{l}\text { Familien- } \\
\text { anamnese }\end{array}$ & $\begin{array}{l}\text { Gesamt-lgE } \\
(\mathrm{kU} / \mathrm{l})\end{array}$ & $\begin{array}{l}\text { Pricktest } \\
\text { Chinchilla }\end{array}$ & $\begin{array}{l}\text { Nasentest } \\
\text { Chinchilla }\end{array}$ & Beschwerden \\
\hline 30 J., w & negativ & 195 & positiv & positiv & ganzjährig RCA \\
\hline $27 \mathrm{~J} ., \mathrm{m}$ & negativ & 285 & positiv & negativ & ganzjährig Asthma \\
\hline 24 J., w & negativ & 291 & positiv & negativ & saisonal RCA \\
\hline $20 \mathrm{~J} ., \mathrm{w}$ & n.b. & 1209 & positiv & positiv & $\begin{array}{l}\text { ganzjährig Asthma und } \\
\text { RCA }\end{array}$ \\
\hline 10 J., w & positiv & Nicht geprüft & positiv & positiv & ganzjährig Asthma \\
\hline $5 \mathrm{J.,} \mathrm{m}$ & negativ & Nicht geprüft & positiv & negativ & $\begin{array}{l}\text { ganzjährig Asthma und } \\
\text { RCA }\end{array}$ \\
\hline
\end{tabular}

Tab. 2 Patientenprofil

RCA = Rhinoconjunctivitis allergica

war bei 4 Patienten negativ. Bei 5 Patienten wurden 1 - 2 Tiere im Wohnzimmer, bei einem 10-jährigen Mädchen im Kinderzimmer gehalten. Alle Tiere hatten regelmäßigen Freilauf im Zimmer. In zwei Familien wurde im Wohnzimmer geraucht (Tab. 2).

Von drei Patienten konnten Informationen zu den Umständen der Anschaffung gesammelt werden. Bei 2 Patienten stand die Nachtaktivität der Tiere im Vordergrund. Sie hatten zum damaligen Zeitpunkt keine bekannten Allergien. In der Familie eines 5-jährigen Jungen war das Chinchilla ein Ersatz für ein Meerschweinchen, dessen Kontakt bei dem Kind zu Hautausschlag geführt hat. Kein Patient wusste von der Möglichkeit der Entstehung einer Chinchillahaarallergie und wurde auch nicht durch Verkäufer darauf hingewiesen.

Bei drei Patienten konnte mittels Nasentest eine klinisch aktuelle Chinchillasensibilisierung nachgewiesen werden. Eine 30-jährige Frau hatte gleichzeitig klinisch stumme Sensibilisierungen gegenüber Hausstaubmilben und Pferdeepithelien. Eine 20-jährige Patientin litt an Pollinose und Bäckerasthma. Das 10-jährige Mädchen hatte neben der Chinchillahaarallergie auch klinisch aktuelle Sensibilisierungen gegenüber Pollen und Meerschweinchenhaaren.

Die Patienten mit negativen Nasentests wiesen eine Pollenallergie bzw. klinisch stumme Sensibilisierungen gegenüber Katzen- und Pferdehaaren auf. Bei einem 5-jährigen Jungen fanden sich keine weiteren Sensibilisierungen.

In 5 Familien wurden Chinchillas nach einem Jahr weiter gehalten. Die Familie des 5-jährigen Jungen hatte inzwischen die Chinchillas abgeschafft und berichtete seitdem über Beschwerdefreiheit.

\section{Schlussfolgerungen}

Sowohl im Kindes- als auch im Erwachsenenalter kann es bei Chinchillakontakt im Haushalt zu Sensibilisierungen mit der Möglichkeit der Entwicklung einer allergischen ChinchillaRhinokonjunktivitis kommen.

Chinchillas sollten bei atopischer Disposition nicht als Haustiere gehalten werden.

Bereits beim Kauf der Tiere sollte eine Aufklärung über das Allergierisiko erfolgen.
Auch bei nachgewiesener Allergie gegen Chinchillahaare ist die Bereitschaft der Patienten, sich von dem Tier zu trennen, gering.

\section{Literatur}

${ }^{1}$ Pletscher M. Die Bedeutung des Haustieres aus der Sicht des Allergologen, Infoblatt Nr. 17 der Arbeitsgemeinschaft Allergiekrankes Kind.

2 Röder-Thiede M. Chinchillas als Heimtiere, GU Tier-Ratgeber, 8. Auflage. Gräfe und Unzer Verlag, 1999

${ }^{3}$ Hoefner HL. Chinchillas. Vet Clin North Am Small Anim Pract 1994; $103-111$

${ }^{4}$ Perzanowski MS, Rönmark E, Nold B, Lundbäck B, Platts-Mills Th. Relevance of allergens from cats and dogs to asthma in the northernmost province of Sweden: Scools as a major site of exposure. J Allergy Clin Immunol 1999; 103: 1018-102

${ }^{5}$ Spitzauer S. Major cat and dog allergens share IgE epitopes. J Allergy Clin Immunol 1997; 99: 100-106

${ }^{6}$ Müsken H. Innenraumallergene: Klinische Bedeutung, Nachweis und Meidung. Pneumologie 1996; 50: 168 - 176

Prof. Dr. K.-C. Bergmann

Allergie- und Asthma-Klinik

An der Martinusquelle 10 33175 Bad Lippspringe 